\title{
Raising the Bar on Rectal Cancer: The National Accreditation Program for Rectal Cancer
}

\section{Katerina $\mathbf{W}^{*}$}

Department of Surgery, Baylor University Medical Center, Dallas, TX, USA

${ }^{*}$ Corresponding author: Katerina Wells, Director of Colorectal Research, Department of Surgery, Baylor University Medical Center, Dallas, TX, USA, Tel: 4698007180; Fax: 6179099171; E-mail: katerina.wells@bswhealth.org

Received date: December 18, 2017; Accepted date: December 23, 2017; Published date: December 27, 2017

Citation: Katerina W (2017) Raising the Bar on Rectal Cancer: The National Accreditation Program for Rectal Cancer. Colorec Cancer. Vol.3 No. 2:17.

Copyright: (c) 2017 Katerina W. This is an open-access article distributed under the terms of the Creative Commons Attribution License, which permits unrestricted use, distribution, and reproduction in any medium, provided the original author and source are credited.

\section{Abstract}

Since the concept of total mesorectal excision (TME), fostered by Heald, for the surgical management of rectal cancer, a new standard for rectal cancer treatment has emerged. Advances in surgical technique, medical and radiation oncology, radiology, and pathologic assessment have made rectal cancer care a complex and shifting environment such that providing the highest standard of care requires expert and current knowledge of treatment options. In order to achieve consistently high standards of care, standardization of the process is needed in the form of the National Accreditation Program for Rectal Cancer (NAPRC). The NAPRC is a newly established program developed with the primary goal of improving rectal cancer outcomes through a multidisciplinary approach. It is the expectation that the NAPRC will, in addition, improve awareness of rectal cancer throughout the medical community as a complex and high-risk condition best treated in specialized centers.

Keywords: National accreditation program; Rectal cancer; Proctectomy; Total mesorectal excision; Centers of excellence

\section{Introduction}

Since the introduction of total mesorectal excision (TME) by Heald, proctectomy for rectal cancer has been considered a complex and high-risk procedure with long term outcomes dependent upon expert surgical technique [1]. TME is now the standard of care for surgical management and studies support that both oncologic and functional outcomes are optimized in a high surgical volume setting [2,3]. Emerging techniques including robotic and transanal TME gain are gaining popularity; however, it is important to keep innovation in the hands of experienced colorectal surgeon to avoid compromise of this surgical standard [4]. As surgical management of rectal cancer has evolved, so too have medical treatment options including variations in neoadjuvant therapy regimens, immunotherapy, and experimental approaches to rectal cancer, including nonoperative management. Additionally, advanced imaging modalities including rectal-cancer protocol pelvic MRI and experienced radiologists to offer interpretation have strengthened the ability to perform more accurate preoperative staging and treatment planning [5].

In an effort to establish evidenced-based diagnostic and treatment protocols in this rapidly advancing field, expertise and research infrastructure to support clinical trials are necessary. Indeed, the management of rectal cancer has become a multidisciplinary effort focused on the goal of better patient outcomes including decreased rates of local recurrence and morbidity, improved survival and functional outcomes as well as more appropriate use of neoadjuvant and adjuvant therapies. The key to maintaining the highest standards of multidisciplinary care in this shifting landscape is accreditation of centers specializing in rectal cancer.

\section{National Accreditation Program for Rectal Cancer (NAPRC)}

The National Accreditation Program for Rectal Cancer (NAPRC) is a newly developed initiative formed in collaboration with The OSTRiCh Consortium (Optimizing the Surgical Treatment of Rectal Cancer) and the Commission on Cancer ( $\mathrm{CoC})$, a quality program of the American College of Surgeons (ACS). The effort is borne out of long-established experience in several European countries where high volume centers of excellence employing a multidisciplinary approach and standardized treatment processes have demonstrated improved oncologic outcomes [6].

The NAPRC was officially announced at the ACS Clinical Congress in San Diego, CA, in October 2017 with the opportunity to apply for accreditation currently available to physician champions in institutions across the United States. The program's foundation is built upon establishment of a multidisciplinary team of qualified physicians and coordinators to provide expert management consensus, patient care processes to optimize the delivery of treatment plans, 
implementation of evidenced based treatment process and protocols, and data collection with internal auditing of compliance to program standards for the purpose of performance improvement [7]. The October 2017 edition NAPRC standards manual is an outline of 22 standards covering the areas of Program Management, Clinical Services, and Quality Improvement. The standards address various topics in rectal cancer care from participants' attendance in multidisciplinary conferences, institutional pathologic review of specimens, preoperative staging with laboratory studies and rectal cancer-protocol MRI, standardized surgical and pathologic technique and reporting, pre- and post- surgical treatment planning, and interval data auditing for Quality Improvement [8] (Table 1).

Table 1 National Accreditation Program for Rectal Cancer Standards [1].

\begin{tabular}{|c|c|c|}
\hline Areas & Standard & Description \\
\hline \multirow{7}{*}{ Program Management } & 1.1 & Commission on Cancer Accreditation \\
\hline & 1.2 & Rectal Cancer Multidisciplinary Care \\
\hline & 1.3 & Rectal Cancer Multidisciplinary Team Attendance \\
\hline & 1.4 & Rectal Cancer Multidisciplinary Team Meetings \\
\hline & 1.5 & Rectal Cancer Program Director \\
\hline & 1.6 & Rectal Cancer Program Coordinator \\
\hline & 1.7 & Rectal Cancer Program Education \\
\hline \multirow{13}{*}{ Clinical Services } & 2.1 & Review of Diagnostic Pathology \\
\hline & 2.2 & Staging before Definitive Treatment \\
\hline & 2.3 & Standardized Staging Reporting for Magnetic Resonance Imaging Results \\
\hline & 2.4 & Carcinoembryonic Antigen Level \\
\hline & 2.5 & Rectal Cancer Multidisciplinary Team Treatment Planning Discussion \\
\hline & 2.6 & Treatment Evaluation and Recommendation Summary \\
\hline & 2.7 & Definitive Treatment Timing \\
\hline & 2.8 & Surgical Resection and Standardized Operative Reporting \\
\hline & 2.9 & Pathology Reports after Surgical Resection \\
\hline & 2.10 & Photographs of Surgical Specimens \\
\hline & 2.11 & Multidisciplinary Team Treatment Outcome Discussion \\
\hline & 2.12 & Treatment Outcome Discussion Summary \\
\hline & 2.13 & Adjuvant Therapy after Surgical Resection \\
\hline \multirow{2}{*}{ Quality Improvement } & 3.1 & Rapid Quality Reporting System \\
\hline & 3.2 & Accountability and Quality Improvement Measures \\
\hline
\end{tabular}

Each standard was devised in collaboration with specialists from contributing disciplines to provide the highest standard of care guidelines available to the practicing physician.

Accreditation is available to institutions that hold Commision on Cancer ( $\mathrm{CoC}$ ) accreditation and have conducted a Rectal Cancer Program (RCP) consistent with NAPRC standards for at least 12 months. Following application for accreditation, survey of the institutional site is performed to evaluate compliance to standards and appropriate clinical outcomes.

\section{Expectations and Goals}

The expectation of the NAPRC is that rectal cancer outcomes will improve and be consistently maintained at the highest possible level in accredited programs. Quality indicators under review include adherence to national standards and guidelines for staging, appropriate neoadjuvant and adjuvant therapies, surgical quality indicators including TME grade, circumferential resection margin, and perioperative morbidity, oncologic outcomes including local recurrence and survival, as well as appropriate genetic counseling [9].

Compliance is stringent approaching $80-95 \%$ for most standards, therefore it is anticipated that accredited programs 
will be pressed to meet treatment standards. As the NAPRC program is newly introduced to US institutions, growing pains will occur in areas where previous practice standards may not match with NAPRC standards.

Ultimately, the primary outcome that the NAPRC will improve oncologic outcomes is required to justify its existence down the road; however, implementation will be a gradual process with advantages in long term outcomes likely taking years to demonstrate. In the meanwhile, the NAPRC has tremendous value in the secondary outcomes to be gained by its presence. Certainly, participation in the CoC is expected to increase, as this is a prerequisite to NAPRC application. Greater participation with the $\mathrm{CoC}$ by centers interested in NAPRC accreditation will increase mandatory reporting and standards for all cancer cases, creating added benefit beyond rectal cancer. In addition, having a formal process for review is, in and of itself, invaluable for critical appraisal of current practice and outcomes and for identifying specific areas of improvement. Real-time auditing of compliance to standards and outcomes accelerates process implementation. Lastly, the presence of NAPRC is impactful to the entire medical community by raising awareness that rectal cancer is a highrisk condition that requires an expert multidisciplinary approach in an accredited center. Such awareness should force referring physicians to seek out these centers and calls surgeons to task to provide this high level of care in their practicing institutions or refer them on to accredited centers.

\section{Conclusion}

Whatever the advances in the management of rectal cancer, defining standards for process evaluation and treatment in a nationally accredited program is the way to ensure the highest level of care for patients. Through OSTRiCh recommendations to the ACS Commission on Cancer ( $\mathrm{CoC})$, the National
Accreditation Program for Rectal Cancer (NAPRC) has now become a reality and centers around the US are called to task to meet this requirement. This highly celebrated program heralds a new and much anticipated horizon in rectal cancer care.

\section{References}

1. Heald RJ, Husband EM, Ryall RD (1982) The mesorectum in rectal cancer surgery-the clue to pelvic recurrence? Br J Surg 69: 613-616.

2. Baek JH, Alrubaie A, Guzman EA, Choi SK, Anderson C, et al. (2012) The association of hospital volume with rectal cancer surgery outcomes. Int J Colorectal Dis 28: 191-196.

3. Aquina $\mathrm{CT}$, Probst $\mathrm{CP}$, Becerra $\mathrm{AZ}$, lannuzzi $\mathrm{JC}$, Kelly $\mathrm{KN}$, et al. (2016) High volume improves outcomes: The argument for centralization of rectal cancer surgery. Surgery 159: 736-748.

4. Marks JH, Frenkel JL, D'Andrea AP, Greenleaf CE (2011) Maximizing rectal cancer results: TEM and TATA techniques to expand sphincter preservation. Surgical Oncology Clinics of NA 20: $501-520$

5. Bhoday J, Balyasnikova S, Wale A, Brown G (2017) How Should Imaging Direct/Orient Management of Rectal Cancer? Clinics in Colon and Rectal Surgery 30: 297-312.

6. Wexner S, Berho M (2015) The long overdue inception of accreditation of centres for rectal cancer surgery in the United States. Colorectal Dis 17: 465-467.

7. https://www.facs.org/quality-programs/cancer/naprc

8. https://www.Facs.org/Quality-Programs/Cancer/Naprc/ Standards.

9. Wexner SD, Berho ME (2017) The Rationale for and Reality of the New National Accreditation Program for Rectal Cancer. Diseases of the Colon \& Rectum 60: 595-602. 